Wilfrid Laurier University

Scholars Commons @ Laurier

Physics and Computer Science Faculty

Publications

Physics and Computer Science

2005

\title{
Ion-Exchanged Glass Waveguides with Low Birefringence for a Broad Range of Waveguide Widths
}

\author{
Sanna Yliniemi \\ University of Arizona \\ Brian R. West \\ Wilfrid Laurier University, bwest@wlu.ca \\ Seppo Honkanen \\ University of Arizona
}

Follow this and additional works at: https://scholars.wlu.ca/phys_faculty

\section{Recommended Citation}

Yliniemi, Sanna; West, Brian R.; and Honkanen, Seppo, "Ion-Exchanged Glass Waveguides with Low Birefringence for a Broad Range of Waveguide Widths" (2005). Physics and Computer Science Faculty Publications. 87.

https://scholars.wlu.ca/phys_faculty/87

This Article is brought to you for free and open access by the Physics and Computer Science at Scholars Commons @ Laurier. It has been accepted for inclusion in Physics and Computer Science Faculty Publications by an authorized administrator of Scholars Commons @ Laurier. For more information, please contact scholarscommons@wlu.ca. 


\title{
lon-exchanged glass waveguides with low birefringence for a broad range of waveguide widths
}

\author{
Sanna Yliniemi, Brian R. West, and Seppo Honkanen
}

\begin{abstract}
Optical communications networks require integrated photonic components with negligible polarization dependence, which typically means that the waveguides must feature very low birefringence. Recent studies have shown that waveguides with low birefringence can be obtained, e.g., by use of silica-onsilicon waveguides or buried ion-exchanged glass waveguides. However, many integrated photonic circuits consist of waveguides with varying widths. Therefore low birefringence is consequently required for waveguides having different widths. This is a difficult task for most waveguide fabrication technologies. We present experimental results on waveguide birefringence for buried silver-sodium ion-exchanged glass waveguides. We show that the waveguide birefringence of the order of $10^{-6}$ for waveguide mask opening widths ranging from 2 to $10 \mu \mathrm{m}$ can be obtained by postprocessing the sample through annealing at an elevated temperature. The measured values are in agreement with the values calculated with our modeling software for ion-exchanged glass waveguides. This unique feature of ion-exchanged waveguides may be of significant importance in a wide variety of integrated photonic circuits requiring polarizationindependent operation. (C) 2005 Optical Society of America
\end{abstract}

OCIS codes: $130.0130,230.7380,260.1440$.

\section{Introduction}

Efficient optical routing is a key requirement to meet the increasing demand for higher system transmission capacities in optical communications networks. Dense wavelength division multiplexing is the leading technology used in optical routing. This technology is implemented with various integrated optical components including add-drop filters and phasedarray wavelength multiplexers and demultiplexers. Planar waveguides used in these components need to feature efficient coupling with fiber, low propagation loss, and negligible birefringence. The first two requirements are obvious from a power consumption point of view; the last requirement results from the fact that the single-mode optical fiber used in longhaul transmission systems is not polarization maintaining. Therefore polarization insensitivity in connecting waveguide components is a crucial requirement to be fulfilled to avoid polarizationdependent performance. However, this is not a

The authors are with the Optical Sciences Center, University of Arizona, Meinel Building, 1630 East University Boulevard, Tucson, Arizona 85721. The e-mail address for S. Yliniemi is syliniemi@optics.arizona.edu.

Received 3 August 2004; accepted 13 October 2004.

0003-6935/05/163358-06\$15.00/0

(C) 2005 Optical Society of America simple task since, unlike fibers, waveguides typically lack cylindrical symmetry, increasing form birefringence. In addition, waveguide fabrication processes induce stresses resulting in birefringence, which is difficult to eliminate.

Several approaches to reduce or compensate for birefringence have been suggested in connection with the leading waveguide technology, silica-on-silicon. These include stress reduction by etching strainrelieving grooves on both sides of the waveguide ${ }^{1,2}$ or by growing additional material layers on top of the waveguide. ${ }^{3,4}$ Albert et al. ${ }^{5}$ showed that waveguide birefringence can be reduced by trimming the waveguide by UV illumination. A similar approach with $\mathrm{CO}_{2}$ laser ablation was suggested by Canning and Aslund. ${ }^{6}$ Integration of birefringencecompensating devices into the chip has also been demonstrated. ${ }^{7}$ The major drawback common in all these methods is that they require additional processing steps and therefore increase the complexity of the device fabrication.

In this paper we study birefringence properties of buried $\mathrm{Ag}^{+}-\mathrm{Na}^{+}$ion-exchanged glass waveguides, the other widely employed glass waveguide technology. Special emphasis is put on the effect of waveguide width on waveguide birefringence, which is of crucial importance in some devices. ${ }^{8}$ This technology was chosen since glass waveguides fabricated 
by ion exchange feature good mode matching with optical fibers, low propagation loss, and low birefringence in general. ${ }^{9-13}$ The processing of ion-exchanged waveguides is simple, and they can be easily integrated with other passive or active waveguide components on glass substrates. We demonstrate that low birefringence, of the order of $10^{-5}$ or below, can be obtained in buried $\mathrm{Ag}^{+}-\mathrm{Na}^{+}$ion-exchanged waveguides for a wide range of waveguide widths. This is in contrast to silica-on-silicon waveguides for which low birefringence is attainable only for a rather limited range of waveguide widths. ${ }^{14}$ To further reduce waveguide birefringence we utilize the feature that, in $\mathrm{Ag}^{+}-\mathrm{Na}^{+}$ion-exchanged waveguides, the stress-induced birefringence can be fully compensated by matching the form birefringence through thermal annealing. The effect of thermal annealing on waveguide birefringence is studied by annealing and characterizing the sample in steps. In Section 2 we describe the ion exchange modeling. In Section 3 we present the ion exchange and burial processes for waveguide fabrication and the annealing process for birefringence reduction. In Section 4 we describe the characterization of the waveguides, and in Section 5 we present the results and discussion. Finally in Section 6 we provide a brief conclusion of our results.

\section{Modeling}

Waveguide birefringence is a sum of form and stressinduced birefringence. In buried silver-sodium ionexchanged waveguides, birefringence from these two sources has opposite signs. Form birefringence favors greater effective refractive index for the quasi-TE mode because the index profile has wider lateral dimensions. This is in contrast to the stress-induced birefringence that favors typically greater effective refractive index for the quasi-TM mode. This is due to the proximity of the glass-air interface that makes it possible for the glass to expand slightly in the direction normal to the surface, thus relaxing the stresses and induced index increase in that direction. By adjusting the form and stress-induced birefringence, zero-birefringence waveguides can be created.

In ion exchange the ions participating in the process have different electronic polarizabilities and different ionic radii and therefore produce a useful refractive-index change between the substrate and the exchanged area. ${ }^{15}$ The differing ionic radii also produce stress that is closely related to the birefringence formation because the thermal expansion experienced by the ion-exchanged glass region and the substrate are different. ${ }^{16} \mathrm{In} \mathrm{Ag}^{+}-\mathrm{Na}^{+}$exchange, compressive stress is produced as the sample cools down since the ionic radius of the silver ion is larger than the ionic radius of the sodium ion. The refractive indices for quasi-TE and quasi-TM modes depend on the stress components $\sigma_{x}, \sigma_{y}$, and $\sigma_{z}$ in the following way ${ }^{16}$ :

$$
\begin{aligned}
& n_{\mathrm{TE}}=n_{0}+C_{1} \sigma_{y}+C_{2}\left(\sigma_{x}+\sigma_{z}\right), \\
& n_{\mathrm{TM}}=n_{0}+C_{1} \sigma_{x}+C_{2}\left(\sigma_{y}+\sigma_{z}\right),
\end{aligned}
$$
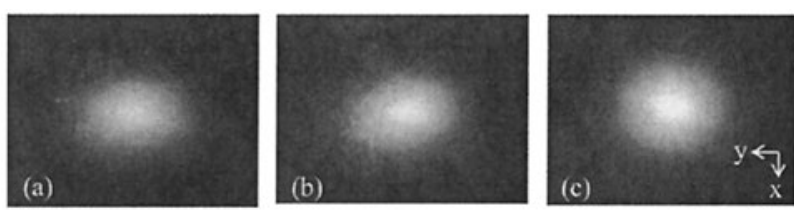

Fig. 1. Mode intensity profiles for waveguides with mask opening widths of (a) $2 \mu \mathrm{m}$, (b) $7 \mu \mathrm{m}$, and (c) for a single-mode fiber.

where $n_{0}$ is the refractive index without stress, and $C_{1}$ and $C_{2}$ are the elasto-optical coefficients $\left(C_{2}>C_{1}\right)$. The $x, y$, and $z$ directions are defined as in Fig. 1 . Since diffusion has a greater extension in the $y$ direction than in the $x$ direction and since the waveguide can slightly expand along the normal to the surface, the stress component in the $x$ direction is smaller than in the $y$ direction $\left(\sigma_{x}<\sigma_{y}\right){ }^{16}$ It can be therefore concluded from Eqs. (1) and (2) that compressive stress favors a larger value for $n_{\mathrm{TM}}$. Exact values for elasto-optical coefficients and stress components in BGG31 glass used in this study are not known, and the measurement of these constants is an elaborate process. Therefore Eqs. (1) and (2) are shown only to qualitatively explain the stress-induced index change in ion-exchanged waveguides, but it is not included in our waveguide modeling.

Modeling of the ion exchange process involves solving for the time evolution of normalized $\mathrm{Ag}^{+}$concentration $C_{\mathrm{Ag}}$,

$$
\begin{aligned}
\frac{\partial C_{\mathrm{Ag}}}{\partial t}= & \frac{D_{\mathrm{Ag}}}{1-(1-M) C_{\mathrm{Ag}}}\left[\nabla^{2} C_{\mathrm{Ag}}+\frac{(1-M)\left(\nabla C_{\mathrm{Ag}}\right)^{2}}{1-(1-M) C_{\mathrm{Ag}}}\right. \\
& \left.-\frac{q \mathbf{E}_{\mathrm{ext}} \cdot \nabla C_{\mathrm{Ag}}}{k T}\right],
\end{aligned}
$$

and is described in detail in Ref. 17. Here, $D_{\mathrm{Ag}}$ and $D_{\mathrm{Na}}$ are the self-diffusion coefficients of silver and sodium ions, respectively, and $M=D_{\mathrm{Ag}} / D_{\mathrm{Na}}$ is their ratio. $\mathbf{E}_{\text {ext }}$ is the applied electric field and $T, k$, and $q$ are the absolute temperature, Boltzmann constant, and the electron charge, respectively. When $M$ differs from unity, $\mathbf{E}_{\text {ext }}$ is nonhomogeneous in the vicinity of the waveguide and must be determined numerically.

Equation (3) is solved numerically by the Peaceman-Rachford alternating direction implicit method, ${ }^{18}$ which combines efficiency and stability over a wide range of processing parameters. After the process has been modeled, a semivectorial finitedifference method $^{19}$ is employed to determine the effective indices of the guided modes. Note that waveguide stress is not accounted for in our model.

\section{Fabrication}

Waveguides with mask opening widths from 2 to $10 \mu \mathrm{m}$ were constructed in BGG31 glass by $\mathrm{Ag}^{+}-\mathrm{Na}^{+}$ ion exchange. Surface channel waveguides fabricated in this way were then buried below the glass surface to reduce propagation losses and to obtain circular waveguides matching well with the fibers. Burial also 


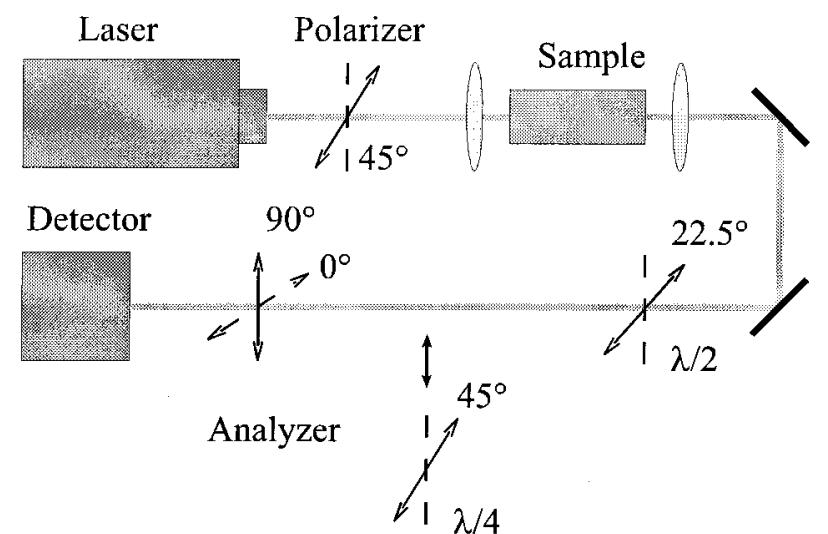

Fig. 2. Measurement setup used to determine the waveguide birefringence of channel waveguides. Linearly polarized light at $\lambda_{0}$ $=1550 \mathrm{~nm}$ is coupled to the sample with a $20 \times$ objective. After the beam emerges from the sample it travels through a $20 \times$ objective, a $\lambda / 2$ plate at an angle of $22.5^{\circ}$, and a rotatable analyzer to the InGaAs detector. The setup also includes a movable $\lambda / 4$ plate at an angle of $45^{\circ}$. All angles are presented with respect to the horizontal axis.

significantly reduces the birefringence by reshaping the index profile.

The process starts with 200-nm-thick titanium layer deposition followed by standard lithography. The titanium layer is used as a diffusion mask during the thermal ion exchange. Ion exchange was performed in a $1: 1 \mathrm{AgNO}_{3}: \mathrm{NaNo}_{3}$ melt at $T=280{ }^{\circ} \mathrm{C}$ for $4500 \mathrm{~s}$. After the ion exchange, the titanium mask was etched away and the waveguides were buried by field-assisted burial. The burial was done for $2400 \mathrm{~s}$ at $T=250{ }^{\circ} \mathrm{C}$ with an applied voltage of $U$ $=320 \mathrm{~V}$ across a 2-mm-thick substrate. Further details of the process have been described in Ref. 20 . With these process parameters, waveguides are buried 5.7-6.8 $\mu \mathrm{m}$ deep in the glass substrate depending on the mask opening width. Mode intensity profiles of the waveguides for two mask opening widths fabricated by $\mathrm{Ag}^{+}-\mathrm{Na}^{+}$ion exchange are presented in Fig. 1 . The mode intensity profile for a single-mode fiber is shown as a comparison. The waveguide mode profiles are almost circular and slightly smaller than the single-mode fiber mode intensity profile as can be seen from Fig. 1. Further reduction of birefringence can be obtained through annealing at an elevated temperature.

\section{Characterization}

We determined the waveguide birefringence using the difference interferometer approach described in Ref. 21. A schematic of the setup is depicted in Fig. 2. First, a linear polarizer at a $45^{\circ}$ angle with respect to the horizontal axis launches orthogonal TE and TM modes with equal amplitudes into the waveguide. Within the waveguide a phase shift $\Delta \Phi$ between the TE and the TM modes is accumulated:

$$
\Delta \Phi=\frac{2 \pi}{\lambda_{0}}\left(n_{\mathrm{TE}}-n_{\mathrm{TM}}\right) L,
$$

where $n_{\mathrm{TE}}$ and $n_{\mathrm{TM}}$ are the effective refractive indices of the TE and TM modes, $L$ is the length of the sample, and $\lambda_{0}$ is the free-space wavelength $\left(\lambda_{0}\right.$ $=1550 \mathrm{~nm}$ ).

After the beam emerges from the output end of the waveguide, the half-wave plate rotates the polarization state by twice the azimuthal angle. The intensities both in the horizontal and in the vertical directions are detected with an InGaAs detector. Next, a quarter-wave plate producing a phase shift of $\pi / 2$ between the TE and the TM modes is added to the setup, and the horizontal and vertical intensities are once again measured. The setup also consists of an infrared camera that is used to check that the laser power is coupled to the mode under study. $\mathrm{Bi}$ refringence values are extracted from the four intensities measured by Jones matrix algebra for polarization states. ${ }^{22}$ Details of the procedure are presented in Appendix A. Since the method described in Appendix A makes no difference between $2 \pi$ multiples, the sample was diced into two pieces with lengths of one third and two thirds of the original sample. Birefringence of both pieces was measured, and the sum birefringence was observed to be equal to the birefringence of the full length sample guaranteeing that no $2 \pi$ multiples were included in the original result.

\section{Results}

Waveguides with mask opening widths of 2 and $3 \mu \mathrm{m}$ were observed to be purely single mode at $1550 \mathrm{~nm}$. Waveguides with mask openings above $3 \mu \mathrm{m}$ were multimode but it was still possible to couple light mainly to the fundamental mode except for the waveguide made with a $10-\mu \mathrm{m}$ mask opening. We measured propagation losses to be below $0.15 \mathrm{~dB}$ for all mask opening widths using the method described in Ref. 23.

The results of the measured and modeled birefringence as a function of a mask opening width are presented in Fig. 3. The measured birefringence is very low, of the order of $10^{-5}$ or below, for all mask opening widths. The accuracy of the measurement was estimated to be $1 \times 10^{-6}$. Birefringence increases linearly as a function of a mask opening width. This results from the fact that the waveguide index profile in wider waveguides is more elliptical, increasing the form birefringence, while the stress-induced birefringence has much smaller dependence on the waveguide width. The modeled birefringence values exhibit the linearly increasing trend as well as a function of a mask opening width. However, the modeled values are slightly higher since the effect of the stress is not included in our model.

The ellipticity of the waveguide index profile can be significantly reduced by thermal annealing. During the annealing, silver ions further diffuse and the index profile becomes more symmetric. The more the sample is annealed, the smaller the form birefringence becomes, and the more dominant the stressinduced birefringence becomes. Since stress-induced 


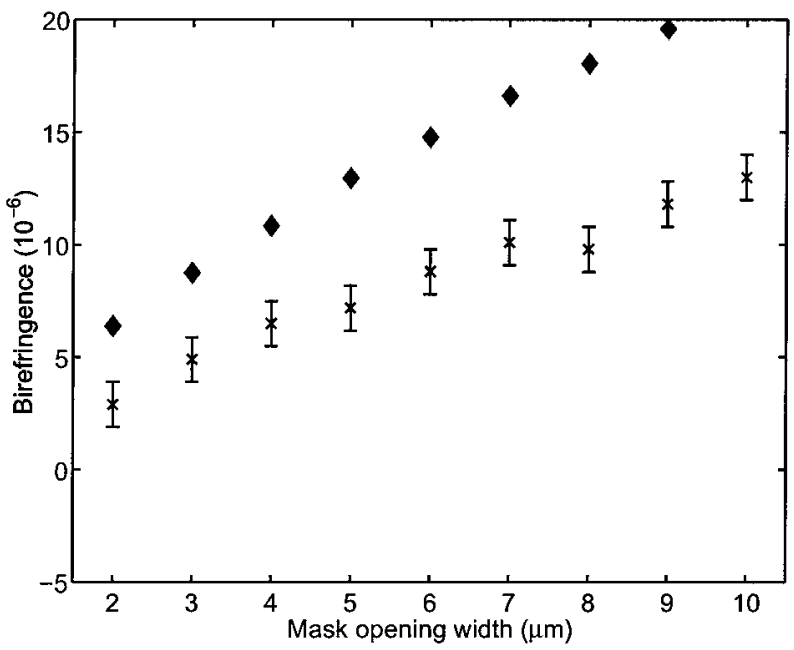

Fig. 3. Measured birefringence $\left(n_{\mathrm{TE}}-n_{\mathrm{TM}}\right)$ after burial (crosses) and the corresponding modeled values (diamonds).

birefringence favors a larger value for $n_{\mathrm{TM}}$, birefringence eventually changes the sign after annealing long enough.

We studied the effect of thermal annealing by placing the sample into the oven at $250{ }^{\circ} \mathrm{C}$ first for $15 \mathrm{~min}$ and then for three successive 30 -min durations. Birefringence was measured after each annealing step. The results are depicted in Fig. 4. After 45-min annealing, birefringence for waveguides with mask opening widths below $4 \mu \mathrm{m}$ is already negative. Also, birefringence for wider waveguides has reduced significantly. By further annealing, birefringence for all the waveguides becomes negative and it starts to saturate to the stress-induced value. It can be concluded that for each waveguide there is a point at which the form and stress-induced birefringence cancel each other and zero net birefringence is produced.

The above experiments suggest that, by annealing the sample long enough, the form birefringence even-

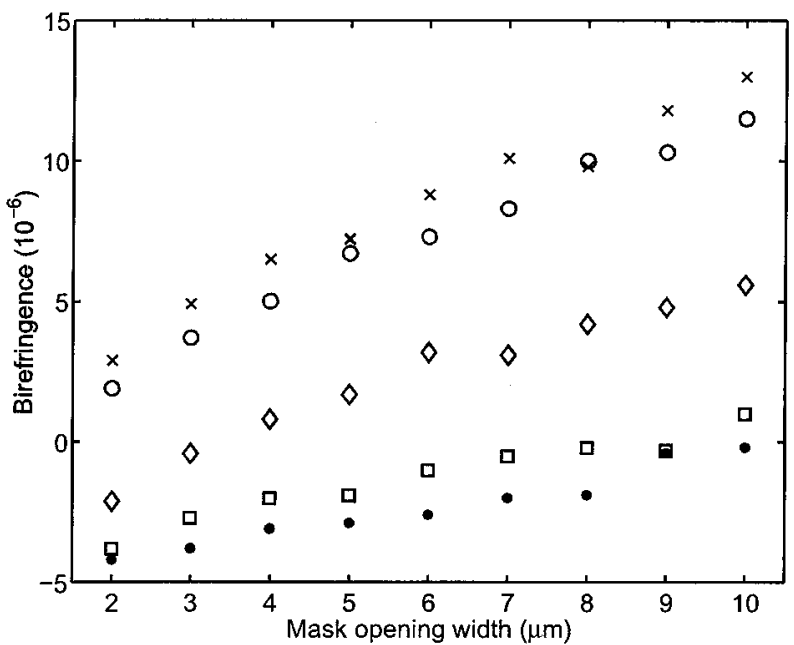

Fig. 4. Birefringence $\left(n_{\mathrm{TE}}-n_{\mathrm{TM}}\right)$ before annealing (crosses) and after annealing at $250{ }^{\circ} \mathrm{C}$ for $15 \mathrm{~min}$ (open circles), $45 \mathrm{~min}$ (diamonds), $75 \mathrm{~min}$ (squares), and $105 \mathrm{~min}$ (filled circles).
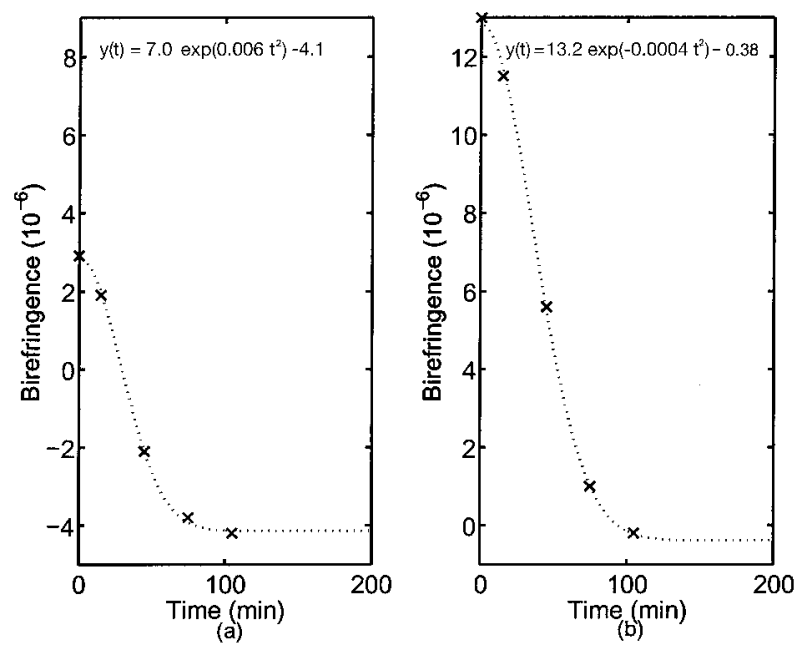

Fig. 5. Exponential fit for birefringence as a function of annealing time for mask opening widths (a) $2 \mu \mathrm{m}$ and (b) $10 \mu \mathrm{m}$.

tually disappears and all that is left is the stressinduced birefringence. Annealing has only a minor effect on the stress-induced birefringence. Because of the nature of the diffusion process, the form birefringence is expected to release according to the function $y(t)=a \exp \left(b t^{2}\right)+c$, where $a, b$, and $c$ are the fitting parameters to be found. To study this statement, the birefringence values obtained after each annealing step were fitted to this function for different mask opening widths. Gaussian fits for the narrowest and the widest waveguide widths are shown in Fig. 5.

The saturation values for birefringence are depicted in Fig. 6. Birefringence has a Gaussian decay for all waveguides and saturates between -4.1 $\times 10^{-6}$ and $-0.38 \times 10^{-6}$ depending on the mask opening width. The results presented in Fig. 6 suggest that stress-induced birefringence is more pronounced for waveguides with narrower lateral dimensions, but the dependence on the waveguide width is not as strong as in the case of form birefrin-

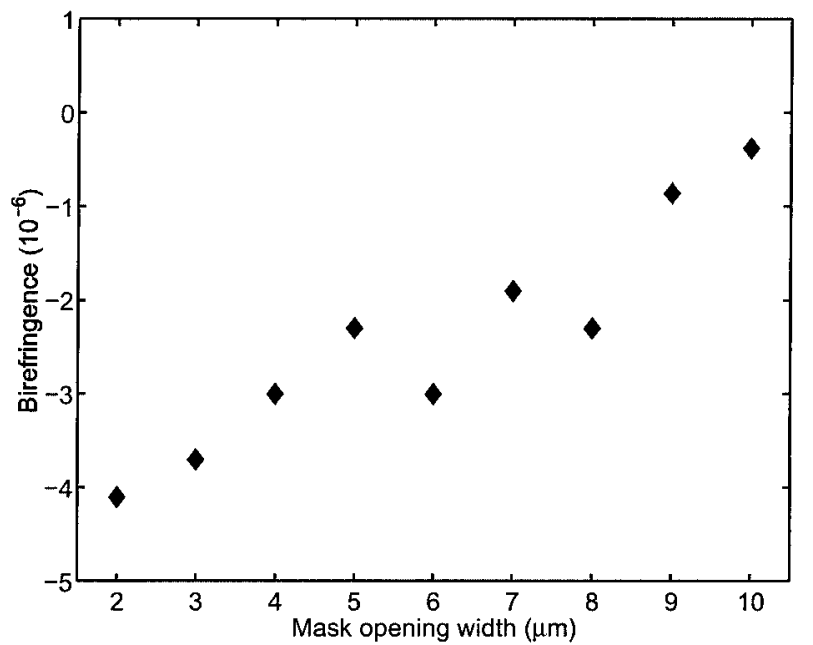

Fig. 6. Saturated birefringence values obtained from the exponential fitting procedure for different mask opening widths. 
gence. However, it is possible that the wider waveguides have not yet reached their saturation values and further annealing would reduce the dependence on waveguide width even more. Small deviations from the linear dependence can be observed for the waveguides with mask opening widths of 6 and $8 \mu \mathrm{m}$. For these two waveguides the saturation value is lower than the one predicted by the general trend by approximately $1 \times 10^{-6}$. Both of the waveguides are multimode, and it is possible that light was not coupled optimally to the fundamental mode during measurements. The uncertainty in the fitting procedure is therefore relatively large due to the accumulated inaccuracy in the subsequent measurements. The fitting procedure would have been more accurate if a shorter annealing step had been used. This was limited by the stability of the annealing oven. Temperature fluctuations in the beginning of each annealing step become more and more pronounced for shorter annealing steps.

\section{Conclusions}

We have demonstrated very low $\left(\sim 10^{-6}\right)$ waveguide birefringence in ion-exchanged glass waveguides for mask opening widths from 2 to $10 \mu \mathrm{m}$. To our knowledge, this is the first time that low birefringence has been presented for such a wide range of waveguide widths. In silica-on-silicon waveguides low birefringence is achieved only for rather restricted

\section{Appendix A: Birefringence Characterization}

We extract the retardance angle $\Delta \Phi$, as defined in Eq. (4), from the data utilizing Jones matrix algebra. ${ }^{2}$ The polarization state of the incident beam entering the sample is

$$
\mathbf{E}^{\text {in }}=\left[\begin{array}{c}
E_{x}^{\text {in }} \\
E_{y}^{\text {in }}
\end{array}\right]=\sqrt{\frac{I_{0}}{2}}\left[\begin{array}{l}
1 \\
1
\end{array}\right],
$$

where $E_{x, y}^{\text {in }}$ are the electric field amplitudes along the $x$ and $y$ directions and $I_{0}$ is the total intensity of the field. The polarization state after the sample and the bare half-wave plate can be presented by the electric field

$$
\mathbf{E}_{1}^{\text {out }}=\mathbf{J}_{\lambda / 2} \mathbf{J}_{s} \mathbf{E}^{\text {in }} .
$$

The polarization state after the sample, half-wave plate, and quarter-wave plate is

$$
\mathbf{E}_{2}^{\text {out }}=\mathbf{J}_{\lambda / 4} \mathbf{J}_{\lambda / 2} \mathbf{J}_{s} \mathbf{E}^{\text {in }} .
$$

In Eqs. (A2) and (A3), $\mathbf{J}_{\lambda / 2}, \mathbf{J}_{\lambda / 4}$, and $\mathbf{J}_{s}$ are the Jones matrices corresponding to the half-wave plate, the quarter-wave plate, and the sample, respectively. The Jones matrix can be represented in a general form as

$$
\mathbf{J}_{i}(\Psi, \Gamma)=\left\{\begin{array}{cc}
\exp [-i(\Gamma / 2)] \cos ^{2} \Psi+\exp [i(\Gamma / 2)] \sin ^{2} \Psi & -i \sin \left(\frac{\Gamma}{2}\right) \sin (2 \Psi) \\
-i \sin \left(\frac{\Gamma}{2}\right) \sin (2 \Psi) & \exp [-i(\Gamma / 2)] \sin ^{2} \Psi+\exp [i(\Gamma / 2)] \cos ^{2} \Psi
\end{array}\right\}
$$

waveguide widths. ${ }^{14}$ Also the birefringence in silicaon-silicon waveguides is usually higher, typically of the order of $10^{-5} .24,25$ This birefringence is low enough for most dense wavelength division multiplexing components, but in many sensor applications birefringence should be even lower, of the order of $10^{-7} .11,12$ In our study, waveguides above a $3-\mu \mathrm{m}$ mask opening width are multimode. This is usually an unfavorable state of matters, but in some applications, like in an add-drop filter presented in Ref. 8, multimode waveguides with low birefringence are specifically needed. The observed birefringence is a result of form and stress-induced birefringence. We were able to reduce significantly the form birefringence by annealing the sample. In addition, it was shown that zero-birefringence waveguides can be created by properly choosing the annealing time. Low birefringence and the consequential minimal polarization-dependent loss are favorable features in many dense wavelength division multiplexing components. where $\Psi$ is the azimuthal angle of the corresponding polarization element and $\Gamma$ is the phase difference caused by the element. The azimuthal angle and the phase retardance for the half- and quarter-wave plates and for the sample are shown in Table 1.

With Eqs. (A2)-(A4), the values for the four intensities at the detector can be presented as

$$
\begin{aligned}
& I_{1 x}=E_{1 x}^{\text {out }} E_{1 x}^{\text {out* }}=I_{0} / 2[1+\cos (\Delta \Phi)], \\
& I_{1 y}=E_{1 y}^{\text {out }} E_{1 y}^{\text {out** }}=I_{0} / 2[1-\cos (\Delta \Phi)], \\
& I_{2 x}=E_{2 x}^{\text {out }} E_{2 x}^{\text {out* }}=I_{0} / 2[1+\sin (\Delta \Phi)], \\
& I_{2 y}=E_{2 y}^{\text {out }} E_{2 y}^{\text {out* }}=I_{0} / 2[1-\sin (\Delta \Phi)] .
\end{aligned}
$$

From these intensities the value for the phase retardance can be derived as

$$
\Delta \Phi=\arctan \left[\frac{\left(I_{1 y}+I_{1 x}\right)\left(I_{2 y}-I_{2 x}\right)}{\left(I_{1 y}-I_{1 x}\right)\left(I_{2 y}-I_{2 x}\right)}\right] .
$$


Table 1. Azimuthal Angle and Phase Shift used in Eq. (A4) for Half- and Quarter-Wave Plates and the Sample

\begin{tabular}{lcc}
\hline Element & $\Psi$ & $\Gamma$ \\
\hline$\lambda / 2$ plate & $22.5^{\circ}$ & $\pi$ \\
$\lambda / 4$ plate & $45^{\circ}$ & $\pi / 2$ \\
Sample & $0^{\circ}$ & $\Delta \Phi$ \\
\hline
\end{tabular}

The value for birefringence, defined as $n_{\mathrm{TE}}-n_{\mathrm{TM}}$, can be calculated from Eqs. (4) and (A9).

We thank Mike Morrell and Jason Auxier for their help with measurements. Support from the Technology and Research Initiative Funding (State of Arizona Photonics Initiative) is appreciated. S. Yliniemi also thanks the Academy of Finland and Magnus Ehrnrooth's foundation for financial support.

\section{References}

1. E. Wildermuth, Ch. Nadler, M. Lanker, W. Hunziker, and H. Melchior, "Penalty-free polarization compensation of $\mathrm{SiO}_{2} / \mathrm{Si}$ arrayed waveguide grating wavelength multiplexers using stress release grooves," Electron. Lett. 34, 1661-1663 (1998).

2. C. K. Nadler, E. B. Wildermuth, M. Lanker, W. Hunziker, and H. Melchior, "Polarization insensitive, low-loss, low-crosstalk wavelength multiplexer modules," IEEE J. Sel. Top. Quantum Electron. 5, 1407-1412 (1999).

3. H. Takahashi, Y. Hibino, Y. Ohmori, and M. Kawachi, "Polarization-insensitive arrayed-waveguide wavelength multiplexer with birefringence compensating film," IEEE Photon. Technol. Lett. 5, 707-709 (1993).

4. M. Okuno, A. Sugita, K. Jinguji, and M. Kawachi, "Birefringence control of silica waveguides on $\mathrm{Si}$ and its application to a polarization-beam splitter/switch," J. Lightwave Technol. 12, 625-633 (1994).

5. J. Albert, F. Bilodeau, D. C. Johnson, K. O. Hill, S. J. Mihailov, D. Stryckman, T. Kitagawa, and Y. Hibino, "Polarisationindependent strong Bragg gratings in planar lightwave circuits," Electron. Lett. 34, 485-486 (1998).

6. J. Canning and M. Åslund, "Compensation of birefringence within integrated optical components using a $\mathrm{CO}_{2}$ laser," Electron. Lett. 35, 812-814 (1999).

7. H. Takahashi, Y. Hibino, and I. Nishi, "Polarizationinsensitive arrayed-waveguide grating wavelength multiplexer on silicon," Opt. Lett. 17, 499-501 (1992).

8. D. F. Geraghty, D. Provenzano, M. Morrell, S. Honkanen, A. Yariv, and N. Peyghambarian, "Ion-exchanged waveguide add/drop filter," Electron. Lett. 37, 829-831 (2001).

9. P. Äyräs, G. Nunzi Conti, S. Honkanen, and N. Peyghambarian, "Birefringence control for ion-exchanged channel glass waveguides," Appl. Opt. 37, 8400-8405 (1998).

10. J. T. A. Carriere, J. A. Frantz, B. R. Youmans, S. Honkanen, and R. K. Kostuk, "Measurement of waveguide birefringence using a ring resonator," IEEE Photon. Technol. Lett. 16, 11341136 (2004)

11. V. Minier, D. Persegol, J. L. Lovato, and A. Kévorkian, "Integrated optical current sensor with low-birefringence optical waveguides," in Optical Fiber Sensors, Vol. 16 of OSA Technical Digest Series, Postconference Edition (Optical Society of America, Washington, D.C., 1997), pp. 104-107.

12. V. Minier, D. Persegol, J. L. Lovato, G. Clauss, and A. Kévorkian, "Low-birefringence optical waveguides for high performance magneto-optic current sensing," in Digest of the Eighth European Conference on Integrated Optics (ECIO) (Optical Society of America, Washington, D.C., 1997), pp. 94-97.

13. S. Yliniemi, B. R. West, T. Aalto, P. Madasamy, N. Peyghambarian, and S. Honkanen, "Buried ion-exchanged glass waveguides featuring low birefringence with a broad range of waveguide widths," in Integrated Optics and Photonic Integrated Circuits, G. C. Righini and S. Honkanen, eds., Proc. SPIE 5451, 558-564 (2004).

14. K. Wörhoff, C. G. H. Roeloffzen, R. M. de Ridder, G. Segno, L. T. H. Hilderink, and A. Driessen, "Tolerance of polarization independent waveguides for communication devices," in Integrated Optics and Photonic Integrated Circuits, G. C. Righini and S. Honkanen, eds., Proc. SPIE 5451, 369-380 (2004).

15. J. Albert, "Ion exchange from salt melts," in Introduction to Glass Integrated Optics, S. I. Najafi, ed. (Artech House, Boston, Mass., 1992), pp. 7-38.

16. A. Brandenburg, "Stress in ion-exchanged glass waveguides," J. Lightwave Technol. LT-4, 1580-1593 (1986).

17. B. R. West, P. Madasamy, N. Peyghambarian, and S. Honkanen, "Accurate modeling of ion-exchanged glass waveguide structures," J. Non-Cryst. Solids 347, 18-26 (2005).

18. D. W. Peaceman and H. H. Rachford, Jr., "The numerical solution of parabolic and elliptic differential equations," J. Soc. Ind. Appl. Math. 3, 28-41 (1955).

19. C. M. Kim and R. V. Ramaswamy, "Modeling of graded-index channel waveguides using nonuniform finite difference method," J. Lightwave Technol. 7, 1581-1589 (1989).

20. P. Madasamy, B. R. West, M. M. Morrell, D. F. Geraghty, S. Honkanen, and N. Peyghambarian, "Buried ion-exchanged glass waveguides: burial depth dependence on the waveguide width," Opt. Lett. 28, 1132-1134 (2003).

21. W. Lukosz and C. Stamm, "Integrated optical interferometer as relative humidity sensor and differential refractometer," Sens. Actuators A 25, 185-188 (1991).

22. A. Yariv, Optical Electronics in Modern Communications, 2nd. ed. (Oxford U. Press, New York, 1997), pp. 17-29.

23. W. J. Wang, S. Honkanen, S. I. Najafi, and A. Tervonen, "Loss characteristics of potassium and silver double-ion-exchanged glass waveguides," J. Appl. Phys. 74, 1529-1533 (1993).

24. H. Ou, "Different index contrast silica-on-silicon waveguides by PECVD," Electron. Lett. 39, 212-213 (2003).

25. A. Kilian, J. Kirchhof, B. Kuhlow, G. Przyrembel, and W. Wischmann, "Birefringence free planar optical waveguide made by flame hydrolysis deposition (FDH) through tailoring of the overcladding," J. Lightwave Technol. 18, 193-198 (2000). 